\title{
Retroperitoneal lymph node dissection for germ cell tumour
}

\author{
Vy Tran $^{1 \#}$, Luke Gibson $^{1 \#}$, Shomik Sengupta ${ }^{1,2,3}$ \\ ${ }^{1}$ Department of Urology, Eastern Health, Box Hill, Victoria, Australia; ${ }^{2}$ Eastern health Clinical School, Monash University, Box Hill, Victoria, \\ Australia; ${ }^{3}$ Department of Surgery, University of Melbourne, Heidelberg, Victoria, Australia \\ Contributions: (I) Conception and design: S Sengupta; (II) Administrative support: S Sengupta; (III) Provision of study material or patients: None; \\ (IV) Collection and assembly of data: V Tran, L Gibson; (V) Data analysis and interpretation: V Tran, L Gibson; (VI) Manuscript writing: All \\ authors; (VII) Final approval of manuscript: All authors. \\ \#These authors contributed equally to this work. \\ Correspondence to: Professor Shomik Sengupta. Eastern Health Clinical School, Level 2, 5 Arnold Street, Box Hill, Victoria 3128, Australia. \\ Email: shomik.sengupta@monash.edu.
}

\begin{abstract}
Retroperitoneal lymph node dissection (RPLND) is an infrequently used, but important part of the management of men with metastatic germ cell tumours. The surgery aims to remove the lymph nodes from the primary retroperitoneal landing site from testicular tumours, usually accomplished by removing tissue surrounding the great vessels using a split-and-roll technique. RPLND may be carried out as a primary surgical procedure for staging or treatment of metastases. More frequently it is undertaken as a follow-up after chemotherapy for a residual mass that may contain viable tumour or teratoma. RPLND is recognised as a major surgery with significant risks of morbidity and complications, particularly loss of ejaculation secondary to damage to hypogastric nerves. In select cases, especially during primary RPLND, nervesparing surgery may help preserve ejaculation, which maybe of importance to the young men usually treated for germ cell tumours. In recent years, the development of minimally invasive approaches have also offered a means for potential improvement in the pain and post-operative recovery from RPLND. We conducted a narrative review of the literature to assess indications for RPLND, along with operative approaches and techniques and related outcomes. The majority of available literature is in the form of relatively small retrospective case series, hence additional research in this area is desirable.
\end{abstract}

Keywords: Retroperitoneal lymph node dissection (RPLND); testicular cancer; germ cell tumour; surgery

Submitted Feb 04, 2020. Accepted for publication Jun 20, 2020.

doi: 10.21037/tau-2019-suc-18

View this article at: http://dx.doi.org/10.21037/tau-2019-suc-18

\section{Introduction}

Testicular cancer is an uncommon malignancy but remains the most common solid organ malignancy in men aged 15-35 years. Long term cure rates are excellent for even advanced stage disease with a multimodal and multidisciplinary management strategy.

Retroperitoneal lymph node dissection (RPLND) forms part of the treatment algorithm for a subset of men with testicular cancer and contributes to the high cure rates that these men can achieve. This article aims to review the indications and variations in surgical technique, as well as review published outcomes.

\section{Indications}

Broadly speaking, RPLND can be undertaken in two distinct settings: primary surgical treatment and the postchemotherapy or salvage setting. While the surgical approach in these cases is similar, the rationale behind the surgery and outcomes may differ.

\section{Primary RPLND}

\section{Clinical stage I disease}

In the management of stage I non seminomatous germ cell tumour (NSGCT), RPLND may be offered following 
orchidectomy as an alternative to surveillance or adjuvant chemotherapy. Up to $30 \%$ of patients with stage 1 disease may have subclinical metastatic disease at the time of presentation. Primary RPLND in this setting offers diagnostic/staging value, in addition to a therapeutic benefit for those with metastatic disease.

Given the highly chemosensitive nature of NSGCT and the excellent results achieved with surveillance or adjuvant chemotherapy, the role for primary RPLND in stage I disease has reduced over time. A randomised controlled trial comparing RPLND with a single course of adjuvant BEP for stage 1 NSGCT demonstrated a $7 \%$ reduction in recurrence at 2 years in favour of the chemotherapy group (1).

Advantages of a primary approach to RPLND include the ability to more routinely offer minimally invasive and nerve sparing techniques, avoidance of chemotherapy toxicity and a less intensive follow up regimen with lower exposure to ionising radiation from surveillance CT imaging. Likewise, in a population at high risk for noncompliance with a surveillance protocol, primary RPLND may offer a safer management strategy.

\section{Clinical stage II disease}

There is a general consensus that for metastatic germ cell tumour, first line therapy should consist of chemotherapy due to the chemosensitive nature of the tumour. The exception to this is stage IIA NSGCT or pure teratoma without tumour marker elevation in which primary RPLND or surveillance could be offered (2). In the case of surveillance, persistence or growth of a retroperitoneal mass without corresponding increase in tumour markers can be an indication for proceeding with RPLND.

\section{Post chemotherapy (Salvage) RPLND}

\section{Seminoma}

A residual mass post chemotherapy for metastatic seminoma requires careful evaluation with imaging. FDG PET has a role in assessing the mass, and has a high negative predictive value, especially in masses $>3 \mathrm{~cm}$ (3). RPLND is rarely required as salvage chemotherapy is the preferred approach for an enlarging or PET avid mass. In the case of RPLND proceeding, it should be performed in a high-volume centre, as the intense fibrosis in these cases complicates the operative approach.

\section{Non seminomatous germ cell tumour}

Following chemotherapy for metastatic NSGCT, resection of a residual mass with normal tumour markers may be required. Although it is recognised that a significant portion of these will contain no viable tumour (40\%), the indication for surgery is that around $50 \%$ do contain teratoma and $10 \%$ viable germ cell tumour (4).

Generally, a residual mass $>1 \mathrm{~cm}$ in size is indication for proceeding to RPLND, while controversy exists for smaller masses. A surveillance protocol may be used as an alternative, however, patients must be counselled that there is a recognised recurrence rate of up to $9 \%$ (5).

The surgical approach should include a bilateral or modified template dissection in addition to resection of the residual mass. Complex masses may require additional intervention including en bloc resection of the kidney, psoas or great vessels with substitution grafting if needed. In these cases, a multidisciplinary approach to surgery is required.

\section{Technique}

\section{Preoperative preparation}

Appropriate perioperative counselling is mandatory as would be expected for any major operation. In a fertile cohort of men, the risks to antegrade ejaculation and the role and success of a nerve sparing procedure must be discussed. Routine perioperative assessment should be performed, including a group and hold or crossmatch. In patients who have received bleomycin containing chemotherapy regimens, preoperative lung function testing and anaesthetic assessment are necessary. Bleomycin induced lung disease can lead to interstitial pneumonitis and fibrosis. Intra-operative management to minimise pulmonary complications include minimising fluid overload and the use of air rather than supplemental oxygen for ventilation.

Large or complex masses or those involving other organs may require additional procedures including nephrectomy and aortic or vena caval resection and substitution. Adequate preparation should include the presence or availability of other specialty surgical units in cases with anticipated difficulties.

\section{RPLND templates}

Various templates have been described for the limits of RPLND, and their use and extent continue to generate controversy in the management of germ cell tumours. Advantages of using template dissections include limiting 
morbidity of the procedure and preserving antegrade ejaculation, however, the main drawback is the potential for inadequate dissection leaving a patient at risk of relapse.

The rationale for modified template dissections is based on the well understood pattern of landing zones of lymphatic drainage from testicular primaries. The decision to pursue a modified template dissection depends on the laterality of the primary along with the presence and size of any retroperitoneal mass.

Data suggest that the use of template dissections in appropriately selected cohorts may offer durable long-term outcomes with a reduction in morbidity when compared with more extensive dissections (6).

\section{Bilateral template}

The described boundaries of the bilateral template dissection include both ureters laterally, renal hilum superiorly and the common iliac arteries inferiorly. Approach is as described below. Nodal packets obtained include paraaortic, interaortocaval, paracaval and gonadal vein ipsilateral to the primary tumour down to the level of the deep inguinal ring.

\section{Right modified template}

Boundaries of right modified template include the ureter laterally, periaortic lymphatics above IMA medially, renal hilum superiorly and right common iliac artery inferiorly.

Exposure is obtained by incising the root of the small bowel mesentery as described above. Nodal packets obtained include paracaval, interaortocaval and right gonadal vein.

\section{Left modified template}

Likewise, boundaries of left modified template include the ureter laterally, periaortic lymphatics above IMA medially, renal hilum superiorly and left common iliac artery. Adequate exposure can be obtained by medial mobilisation of the left colon.

Nodal packets removed include the para-aortic and left gonadal vein.

\section{Open RPLND}

The patient is placed in the supine position and a midline laparotomy is performed, with division of the falciform ligament. Exposure is achieved with a selfretaining retractor such as the Omnitract system. Rarely, a thoracoabdominal approach may be used for large or complex masses with anticipated difficulties.

The retroperitoneum can be accessed by incising the posterior peritoneum along the root of the small bowel mesentery from caecum to ligament of Treitz. For larger masses, the incision can continue around caecum and along the line of Toldt to adequately mobilise the right colon. Mobilisation of the left colon medially may be required for some cases with large para-aortic masses.

The "split and roll" technique is generally used over the great vessels to safely identify critical branches and tributaries. The optimal plane of dissection is on the adventitia of the great vessels, which minimises the risks of positive margins on tumour or inadvertent vascular damage. Dissection commences over the aorta inferior to the crossings of the left renal vein, then is continued caudally to identify the origin of the inferior mesenteric artery (IMA). The IMA is usually preserved in right modified template dissection but may be required to be sacrificed in bilateral dissection to obtain adequate exposure. Ligation of larger lymphatic channels should be performed throughout to reduce the risk of postoperative chyle leak.

Lymphatics overlying the aorta are rolled medially to identify the right lumbar arteries, which are ligated to access to the interaortocaval packet. Likewise, the tissue overlying the aorta is rolled laterally to identify and ligate the left lumbar arteries to obtain complete vascular control of the abdominal aorta below the renal hilum. The ligation of lumbar arteries does incur the risk of devascularisation of the spinal cord with resultant paraplegia but is considered essential for complete clearance of the retro-aortic lymph nodes.

A similar split and roll technique is used over the IVC commencing at the level of the right renal hilum, then carried caudally. The origin of the right gonadal vein and the highly variable lumbar veins need to be identified, all of which are ligated to obtain vascular control of the IVC.

Following adequate exposure and vascular control, the required lymph node packets can then be harvested. Posterior control of the lumbar veins and arteries is required as the nodal packets are dissected free from the vertebral ligaments and muscles. Care must be taken to protect the sympathetic trunk during this dissection.

\section{Nerve sparing technique}

Nerve sparing RPLND focusses on preservation of the hypogastric plexus and its branches, which course over the anterior aorta and aortic bifurcation. Nerve sparing can 
preserve ejaculatory function in $99-100 \%$ of patients.

The left sympathetic chain is identified running laterally to vertebral column. Individual post ganglionic fibres are identified exiting the sympathetic chain and are sharply dissected and elevated with vessel loops. The right sided postganglionic fibres are identified emerging from the lateral surface of the IVC in the interaortocaval region and are likewise dissected and slung with vessel loops. The lymphatic tissue remaining below the identified nerves is then removed in a standard fashion to complete the dissection (7).

Nerve sparing in the post chemotherapy setting becomes more difficult due to fibrosis of tumour and surrounding tissue but may still be possible for those with limited disease. Alternatively, a selective or unilateral nerve spare may be performed, although rates of ejaculatory preservation are lower compared to bilateral nerve sparing. For cases in which the resectability of tumour is in doubt, the primary oncological outcome of the operation must not be compromised in attempting nerve preservation

\section{Minimally invasive RPLND}

Traditionally RPLND was routinely performed in an open fashion, however, in recent years the development of minimally invasive options has created the option of a less morbid procedure. Given the oncological principles underlying the procedure, it is critically important that the quality and extent of lymph node dissection is not compromised in striving for a minimally invasive approach. When minimally invasive approaches were developed initially, a diagnostic or staging approach was taken due to the technical difficulties of the procedure. However, in contemporary series, a therapeutic approach is pursued, with the aim to match the extent of nodal dissection seen with an open approach.

Laparoscopic RPLND (L-RPLND) was first described in the 1990's (8) and was taken up in high volume centres around the world. Retrospective series suggested a benefit of shorter hospital stay and faster return to normal activities when compared with open approach, along with high rates of preservation of antegrade ejaculation (95-100\%; Table 1) (32).
Introduction of robotic-assisted RPLND (RA-RPLND) followed in the 2000s with the implementation of robotics in other areas of urologic oncology, offering potential advantages compared to laparoscopic techniques for a technically demanding operation (33). Safety and feasibility have been demonstrated with the publication of several case series $(28,29)$.

Case series of L-RPLND suggest a comparable staging accuracy and relapse rate to open RPLND in stage I disease, however, no randomised comparisons exist (Table 1). Series of RA-RPLND are mostly limited to small numbers confirming safety and feasibility. The use of minimally invasive approaches in stage II or post chemotherapy setting has been investigated in a variety of small case series, confirming feasibility. Follow up in these series is short, requiring larger series with longer follow up to further investigate oncologic outcomes. It is likely that surgeon expertise will limit the utilisation of minimally invasive approaches to this relatively rare procedure.

\section{Post-operative care}

Routine post-operative care in a high dependency unit is recommended. Fluid administration should be limited in patients who have received bleomycin chemotherapy preoperatively. Mechanical and chemical DVT prophylaxis is mandatory in addition to early mobilisation as appropriate. Generally a clear fluid diet is commenced initially and upgraded as tolerated.

\section{Conclusions}

RPLND forms an integral part of the management of a subset of men with metastatic germ cell tumours. While historically, the open approach carried a significant burden of morbidity, newer, minimally invasive approaches have aimed to reduce morbidity while still achieving the oncological clearance needed. Regardless of the approach used, oncological principles should be adhered to. These men should be managed in a multidisciplinary fashion and preferably in high volume centres to achieve best possible outcomes. 


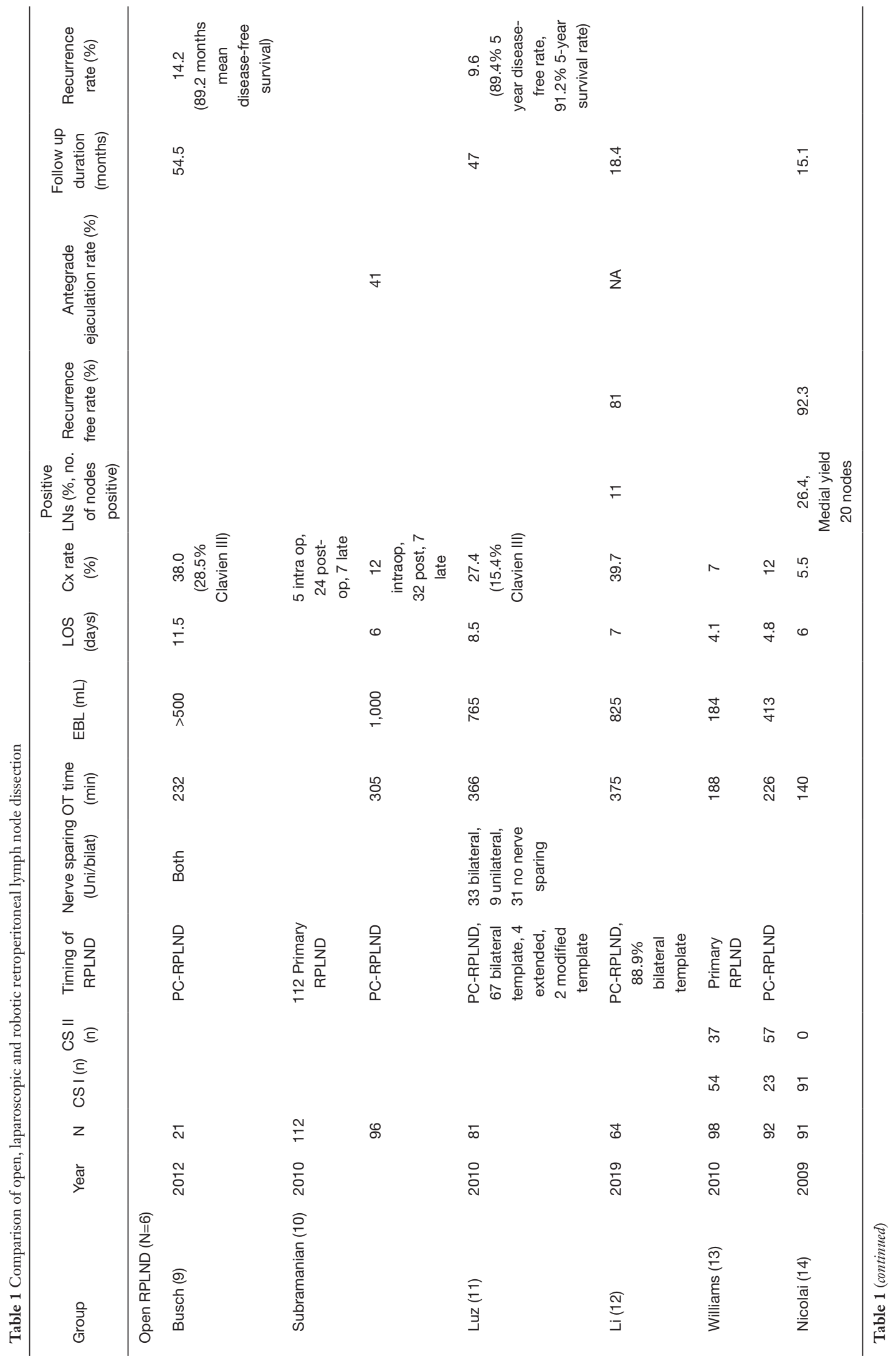




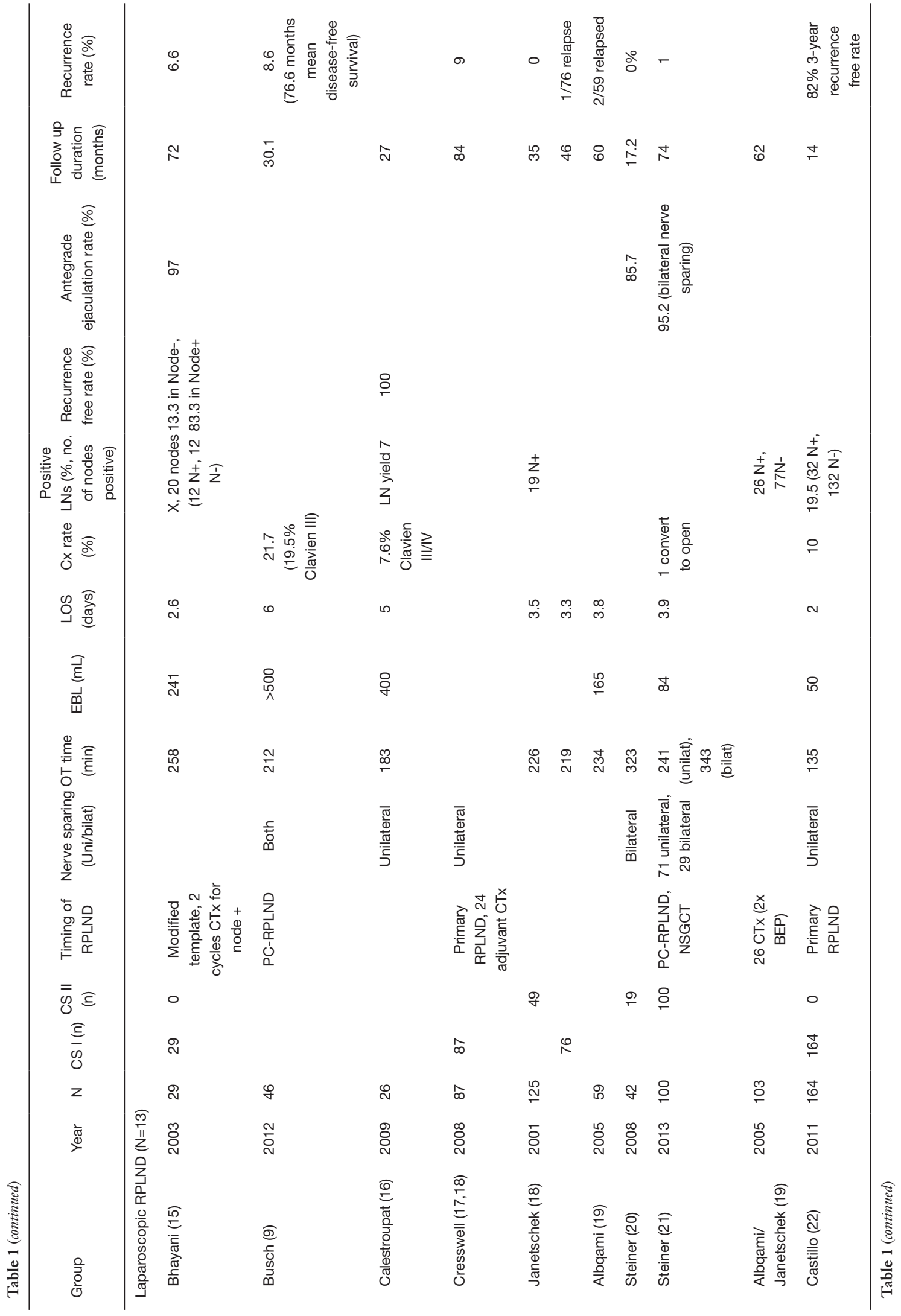




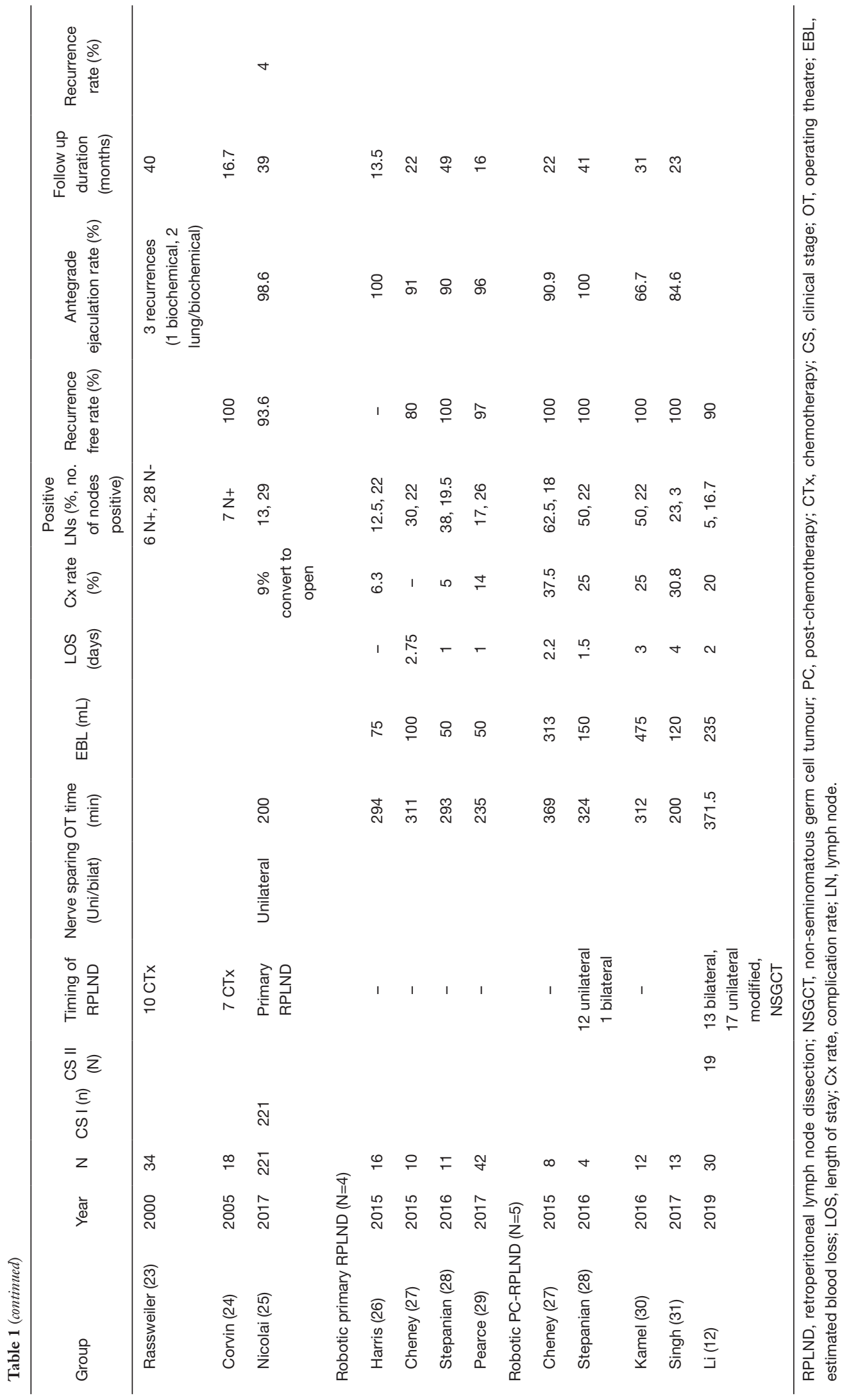




\section{Acknowledgments}

Funding: None.

\section{Footnote}

Provenance and Peer Review: This article was commissioned by the editorial office, Translational Andrology and Urology for the series "Surgery for Urologic Cancers". The article has undergone external peer review.

Conflicts of Interest: All authors have completed the ICMJE uniform disclosure form (available at http://dx.doi. org/10.21037/tau-2019-suc-18). The series "Surgery for Urologic Cancers" was commissioned by the editorial office without any funding or sponsorship. SS served as the unpaid Guest Editor of the series and serves as an unpaid editorial board member of Translational Andrology and Urology from Jul 2018 to Jun 2020. The authors have no other conflicts of interest to declare.

Ethical Statement: The authors are accountable for all aspects of the work in ensuring that questions related to the accuracy or integrity of any part of the work are appropriately investigated and resolved.

Open Access Statement: This is an Open Access article distributed in accordance with the Creative Commons Attribution-NonCommercial-NoDerivs 4.0 International License (CC BY-NC-ND 4.0), which permits the noncommercial replication and distribution of the article with the strict proviso that no changes or edits are made and the original work is properly cited (including links to both the formal publication through the relevant DOI and the license). See: https://creativecommons.org/licenses/by-nc-nd/4.0/.

\section{References}

1. Albers P, Siener R, Krege S, et al. Randomized phase III trial comparing retroperitoneal lymph node dissection with one course of bleomycin and etoposide plus cisplatin chemotherapy in the adjuvant treatment of clinical stage I Nonseminomatous testicular germ cell tumors: AUO trial AH 01/94 by the German Testicular Cancer Study Group. J Clin Oncol 2008;26:2966-72.

2. Albers $\mathrm{P}$, Albrecht $W$, Algaba F, et al. EAU guidelines on testicular cancer: 2011 update. Eur Urol 2011;60:304-19.

3. Treglia G, Sadeghi R, Annunziata S, et al. Diagnostic performance of fluorine-18-fluorodeoxyglucose positron emission tomography in the postchemotherapy management of patients with seminoma: systematic review and meta-analysis. Biomed Res Int 2014;2014:852681.

4. Carver BS, Serio AM, Bajorin D, et al. Improved clinical outcome in recent years for men with metastatic nonseminomatous germ cell tumors. J Clin Oncol 2007;25:5603-8.

5. Ehrlich Y, Brames MJ, Beck SD, et al. Long-term followup of Cisplatin combination chemotherapy in patients with disseminated nonseminomatous germ cell tumors: is a postchemotherapy retroperitoneal lymph node dissection needed after complete remission? J Clin Oncol 2010;28:531-6.

6. Cho JS, Kaimakliotis HZ, Cary C, et al. Modified retroperitoneal lymph node dissection for postchemotherapy residual tumour: a long-term update. BJU Int 2017;120:104-8.

7. Smith JA, Howards SS, Preminger GM, et al. Hinman's atlas of urologic surgery. 3rd ed. Philadelphia: Elsevier/ Saunders; 2012. xxix, 1151 p.

8. Rukstalis DB, Gerber GS, Chodak GW. The application of laparoscopy to retroperitoneal surgery in urology. Arch Esp Urol 1993;46:577-80.

9. Busch J, Magheli A, Erber B, et al. Laparoscopic and open postchemotherapy retroperitoneal lymph node dissection in patients with advanced testicular cancer--a single center analysis. BMC Urol 2012;12:15.

10. Subramanian VS, Nguyen CT, Stephenson AJ, et al. Complications of open primary and post-chemotherapy retroperitoneal lymph node dissection for testicular cancer. Urol Oncol 2010;28:504-9.

11. Luz MA, Kotb AF, Aldousari S, et al. Retroperitoneal lymph node dissection for residual masses after chemotherapy in nonseminomatous germ cell testicular tumor. World J Surg Oncol 2010;8:97.

12. Li R, Duplisea JJ, Petros FG, et al. Robotic

Postchemotherapy Retroperitoneal Lymph Node

Dissection for Testicular Cancer. Eur Urol Oncol. 2019. [Epub ahead of print].

13. Williams SB, McDermott DW, Winston D, et al. Morbidity of open retroperitoneal lymph node dissection for testicular cancer: contemporary perioperative data. BJU Int 2010;105:918-21.

14. Nicolai N, Biasoni D, Aguilera JP, et al. Open versus laparoscopic retroperitoneal lymph node dissection (RPLND) in clinical stage I nonseminomatous germ-cell tumors (NSGCTs): Two contemporary series from a single 
institution. J Clin Oncol 2009;27:5084.

15. Bhayani SB, Ong A, Oh WK, et al. Laparoscopic retroperitoneal lymph node dissection for clinical stage I nonseminomatous germ cell testicular cancer: a long-term update. Urology 2003;62:324-7.

16. Calestroupat JP, Sanchez-Salas R, Cathelineau X, et al. Postchemotherapy laparoscopic retroperitoneal lymph node dissection in nonseminomatous germ-cell tumor. J Endourol 2009;23:645-50.

17. Cresswell J, Scheitlin W, Gozen A, et al. Laparoscopic retroperitoneal lymph node dissection combined with adjuvant chemotherapy for pathological stage II disease in nonseminomatous germ cell tumours: a 15-year experience. BJU Int 2008;102:844-8.

18. Janetschek G, Peschel R, Hobisch A, et al. Laparoscopic retroperitoneal lymph node dissection. J Endourol 2001;15:449-53; discussion 453-5.

19. Albqami N, Janetschek G. Laparoscopic retroperitoneal lymph-node dissection in the management of clinical stage I and II testicular cancer. J Endourol 2005;19:683-92; discussion 692.

20. Steiner H, Zangerl F, Stohr B, et al. Results of bilateral nerve sparing laparoscopic retroperitoneal lymph node dissection for testicular cancer. J Urol 2008;180:1348-52; discussion 1352-3.

21. Steiner H, Leonhartsberger N, Stoehr B, et al. Postchemotherapy laparoscopic retroperitoneal lymph node dissection for low-volume, stage II, nonseminomatous germ cell tumor: first 100 patients. Eur Urol 2013;63:1013-7.

22. Castillo OA, Sanchez-Salas R, Secin FP, et al. Primary laparoscopic retroperitoneal lymph node dissection for clinical stage I nonseminomatous germ-cell testis tumor. Actas Urol Esp 2011;35:22-8.

23. Rassweiler JJ, Frede T, Lenz E, et al. Long-term experience with laparoscopic retroperitoneal lymph node dissection in the management of low-stage testis cancer. Eur Urol 2000;37:251-60.

24. Corvin S, Sturm W, Kuczyk M, et al. Laparoscopic

Cite this article as: Tran V, Gibson L, Sengupta S. Retroperitoneal lymph node dissection for germ cell tumour. Transl Androl Urol 2020;9(6):3103-3111. doi: 10.21037/tau-2019suc-18 retroperitoneal lymphadenectomy in the management of low-stage testicular cancer: technique and results. Minim Invasive Ther Allied Technol 2005;14:52-6.

25. Nicolai N, Tarabelloni N, Gasperoni F, et al. Laparoscopic Retroperitoneal Lymph Node Dissection for Clinical Stage I Nonseminomatous Germ Cell Tumors of the Testis: Safety and Efficacy Analyses at a High Volume Center. J Urol 2018;199:741-7.

26. Harris KT, Gorin MA, Ball MW, et al. A comparative analysis of robotic vs laparoscopic retroperitoneal lymph node dissection for testicular cancer. BJU Int 2015;116:920-3.

27. Cheney SM, Andrews PE, Leibovich BC, et al. Robotassisted retroperitoneal lymph node dissection: technique and initial case series of 18 patients. BJU Int 2015;115:114-20.

28. Stepanian S, Patel M, Porter J. Robot-assisted Laparoscopic Retroperitoneal Lymph Node Dissection for Testicular Cancer: Evolution of the Technique. Eur Urol 2016;70:661-7.

29. Pearce SM, Golan S, Gorin MA, et al. Safety and Early Oncologic Effectiveness of Primary Robotic Retroperitoneal Lymph Node Dissection for Nonseminomatous Germ Cell Testicular Cancer. Eur Urol 2017;71:476-82.

30. Kamel MH, Littlejohn N, Cox M, et al. PostChemotherapy Robotic Retroperitoneal Lymph Node Dissection: Institutional Experience. J Endourol 2016;30:510-9.

31. Singh A, Chatterjee S, Bansal P, et al. Robot-assisted retroperitoneal lymph node dissection: Feasibility and outcome in postchemotherapy residual mass in testicular cancer. Indian J Urol 2017;33:304-9.

32. Gerber GS, Bissada NK, Hulbert JC, et al. Laparoscopic retroperitoneal lymphadenectomy: multi-institutional analysis. J Urol 1994;152:1188-91; discussion 1191-2.

33. Davol P, Sumfest J, Rukstalis D. Robotic-assisted laparoscopic retroperitoneal lymph node dissection. Urology 2006;67:199. 